\title{
Erratum to: Influence of hydrogen on mechanical properties of pure titanium T40 (grade 2) and TA6V ELI (grade 23): a local approach of fracture
}

\author{
Alexandre POLONI ${ }^{*}{ }^{1,2}$, Abdelali OUDRISS ${ }^{1}$, Juan CREUS $^{1}$, Stéphane COHENDOZ ${ }^{1}$, Jamaa BOUHATATE ${ }^{1}$, Simon \\ FRAPPART $^{2}$, Thierry MILLOT ${ }^{2}$, Aude MATHIS $^{2}$, Xavier FEAUGAS ${ }^{1}$ \\ ${ }^{1}$ Laboratoire des Sciences de l'Ingénieur pour l'Environnement UMR-CNRS 7356, Université de La Rochelle, Avenue Michel \\ Crépeau, 17042 La Rochelle, France
}

${ }^{2}$ Naval Group, Technocampus Océan, 5 rue de l'Halbrane, 44340 Bouguenais, France

Original article:

MATEC Web of Conferences

Volume 321, 2020

The $14^{\text {th }}$ World Conference on Titanium (Ti 2019)

https://doi.org/10.1051/matecconf/202032109004

The three following points have been updated and sent to The 14th World Conference on Titanium (Ti 2019) for initial publication, so it should be sufficient to take the model they have actually online instead of the one they've send to MATEC Web Conf.

- The figures 4, 6 and 7 should be updated with the one sent to The 14th World Conference on Titanium (Ti 2019) for initial publication (scales added on macroscales fractures).

- The title of Figure 5, actually: "Hydride layer on a charged mechanical sample of grade 2 (collected perpendicular to the rolling direction) by SEM (a) on the fracture surface (with the fracture initiation) and (b) by a longitudinal cut." Should be replaced by: "Hydride layer on a charged mechanical sample of grade 2 (collected perpendicular to the rolling direction) by SEM $(\mathrm{a}, \mathrm{b})$ on the fracture surface (with the fracture initiation) and (c) by a longitudinal cut."

- The title of Figure 9, actually: "Fracture in intergranular hydrides observed at $1 \mathrm{~mm}$ depth and $150 \mu \mathrm{m}$ below the fracture of a smooth sample with $636 \mathrm{wppm}$ of hydrogen." Should be replaced by: "Fracture in intergranular hydrides observed at $1 \mathrm{~mm}$ depth and $150 \mu \mathrm{m}$ below the fracture of a smooth sample of grade 23 with $636 \mathrm{wppm}$ of hydrogen."

Finally:

- In "Materials end experiments", the text: "iron inclusions $(<0,3 \%$ weight)" should be replaced by: "TiFe precipitates $(\mathrm{Fe}<0,3 \%$ weight in grade 2$)$ "

- Lines should be passed before the Figure 7, which is separated on two pages. It should be on one page for the understanding. Following texts and figures has to be verified and organized accordingly if necessary (if it separates after the modification). 


\title{
Influence of hydrogen on mechanical properties of pure titanium T40 (grade 2) and TA6V ELI (grade 23):
}

\section{a local approach of fracture}

\author{
Alexandre POLONI ${ }^{1,2}$, Abdelali OUDRISS ${ }^{* 1,}$ Juan CREUS ${ }^{1}$, Stéphane COHENDOZ ${ }^{1}$, Jamaa Bouhattate ${ }^{1}$, Simon \\ FRAPPART $^{2}$, Thierry MILLOT ${ }^{2}$, Aude MATHIS $^{2}$, Xavier FEAUGAS $^{1}$
}

alexandre.poloni@univ-lr.fr

${ }^{1}$ Laboratoire des Sciences de l'Ingénieur pour l'Environnement UMR-CNRS 7356, Université de La Rochelle, Avenue Michel Crépeau, 17042 La Rochelle, France

${ }^{2}$ Naval Group, Technocampus Océan, 5 rue de l'Halbrane, 44340 Bouguenais, France

\begin{abstract}
$\underline{\text { Abstract }}$
The effect of hydrogen charging by cathodic polarization on T40 (grade 2) and TA6V ELI (grade 23) in artificial seawater appeared to be dependent on the metallurgical structure of the alloys. Mechanical tensile tests were performed on smooth samples and with different notches without and with hydrogen charging. Evolution of the fracture mode has been studied and the impact of hydrides was questioned. FEM calculation offers the opportunity to associate the local hydrostatic stress $\sigma_{\mathrm{m}}$ and equivalent plastic strain $\varepsilon_{\mathrm{peq}}$ leading to the fracture and to illustrate the evolution of these conditions with hydrogen absorption and hydrides formation. Hydrogen charged by cathodic polarization appeared to have a small impact on grade 2 reducing its $\mathrm{A} \%$, whereas it leads to a strong embrittlement of grade 23 when the solubility limit of $\beta$-phase is exceeded and hydrides formed.
\end{abstract}

\section{Introduction}

In the naval industry, some components are constituted by the assembly of several materials including titanium alloys. These latter have been chosen for their ability to protect themselves against corrosion in seawater [1]. However, to protect the other components, cathodic protection $(\mathrm{CP})$ is applied. If this process is very effective and prevents corrosion, it remains a probable source of hydrogen, which can be absorbed by titanium and induced the embrittlement of the structure. This latter characterized by a loss of mechanical properties and the change of the damage mechanism from ductile to brittle. The main evoked hydrogen-assisted damage mechanism in titanium alloys is the formation of hydrides, however, this mechanism is mainly mentioned when the limit of hydrogen solubility is exceeded which leads to the formation of hydrides [2]. This does not exclude that other mechanisms may also play a role in the embrittlement, and more particularly when the structure is mechanically stressed, and the titanium alloy has a particular metallurgy (phases, textures ...) $[3,4]$. So it is necessary to study the influence of the metallurgical states and the hydrogen concentrations on the mechanical behaviour of titanium alloys, this then requires a better understanding of the contribution of several conditions of $\mathrm{CP}$ (hydrogen concentrations below the solubility and until hydrides formation) on the mechanisms of fracture.

\section{Materials and experiments}

Two materials were studied, the commercially pure grade 2 and the grade 23 titanium alloy. Grade 2 was received in the form of $3 \mathrm{~mm}$-thick rolled sheet from TIMET and grade 23 in the form of $6 \mathrm{~mm}$-thick rolled sheet from AUBERT \& DUVAL. Grade 2, presents a single $\alpha$-phase equiaxed structure with grains measuring $46 \pm 10 \mu \mathrm{m}$ and some intergranular TiFe precipitates ( $\mathrm{Fe}<0,3 \%$ weight in grade 2$)$. The texture analysis shows a preferential 
crystallographic orientation of the basal (0002) pole (Figure 1). The axes are perpendicular to the rolling direction (RD) with the basal pole rotated about $35^{\circ}$ toward the transverse direction (TD). Grains show strong misorientations $\left(>15^{\circ}\right)$. Additionally, it has been observed by TEM only a few dislocations in $\alpha$-grains. Grade 23 presents a $\alpha / \beta$ bimodal structure with a volume fraction of $\beta$-phase about $6 \%$. Primary $\alpha$ grains measure $22 \pm 5$ $\mu \mathrm{m}$, secondary $\alpha$ grains measure $9 \pm 5 \mu \mathrm{m}$ and $\beta$ grains measure approximately $1 \mu \mathrm{m}$. Macro-zones of 100 to 150 $\mu \mathrm{m}$-thick are stacked all along the normal direction (ND) (Figure 1). The dislocation density in the $\alpha$-phase is on the order of $9.410^{13} \mathrm{~m}^{-2}$ and TEM observations highlight dislocation sub-boundaries.

Five types of axisymmetric mechanical samples were used for tensile tests (speed $=10^{-5} \cdot \mathrm{s}^{-1}$ ). A smooth one, two U-notches (U5 with a $5 \mathrm{~mm}$ notch radius and a Kt of 1.3; U3 with a $3 \mathrm{~mm}$ notch radius and a $\mathrm{Kt}$ of 1.45) and two $\mathrm{V}$-notches (V0.25 with a $0.25 \mathrm{~mm}$ notch radius and a Kt of 3.51 ; V0.14 with a $0.14 \mathrm{~mm}$ notch radius and a $\mathrm{Kt}$ of 4.41). Grade 2 samples were collected perpendicular to the rolling direction and grade 23 ones were collected parallel to the rolling direction. Stress axes are TD and RD respectively for grade 2 and grade 23. Hydrogen charging conditions of mechanical samples by cathodic polarization were performed on their gorge length in an artificial seawater $\mathrm{pH} 8$, ASTM-D1141 solution at $30^{\circ} \mathrm{C}$ with a -1,8V/SCE potential, (Figure 2). Tensile tests were performed within 30 minutes after the end of the hydrogen charging to minimize the hydrogen desorption. Hydrogen concentration was measured by thermo-desorption spectroscopy (TDS Horiba EMGA Serie) by collecting a $1 \mathrm{~mm}$-thick slice in the un-necked zone of mechanical samples after tensile tests. Fractures were analyzed by SEM (JEOL JSM 5410 LV). Then, transversal surfaces were polished until OPS Master-Met vibropolishing and observed by SEM (FEI Quanta 200 ESEM/FEG with EBSD TSL/EDAX). As the last step, local mechanical properties (hydrostatic stress and equivalent plastic strain) were identified by calculations using the Finite Element Method (FEM) with an elasto-plastic isotropic law.

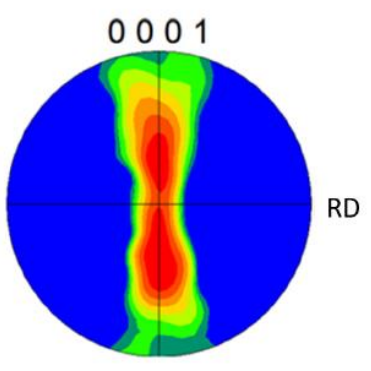

ND

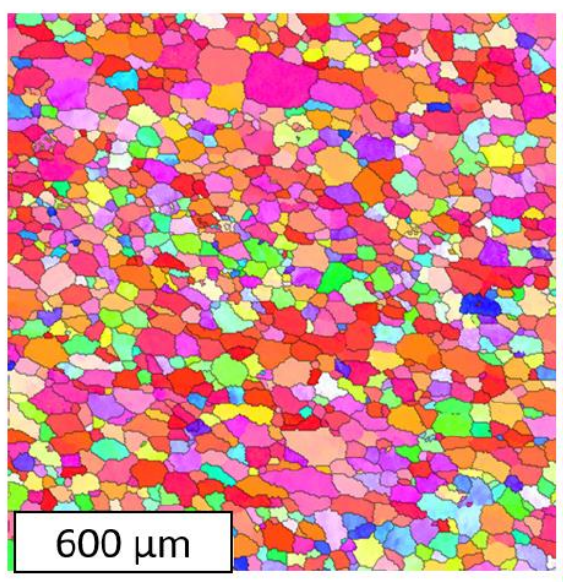

(b)

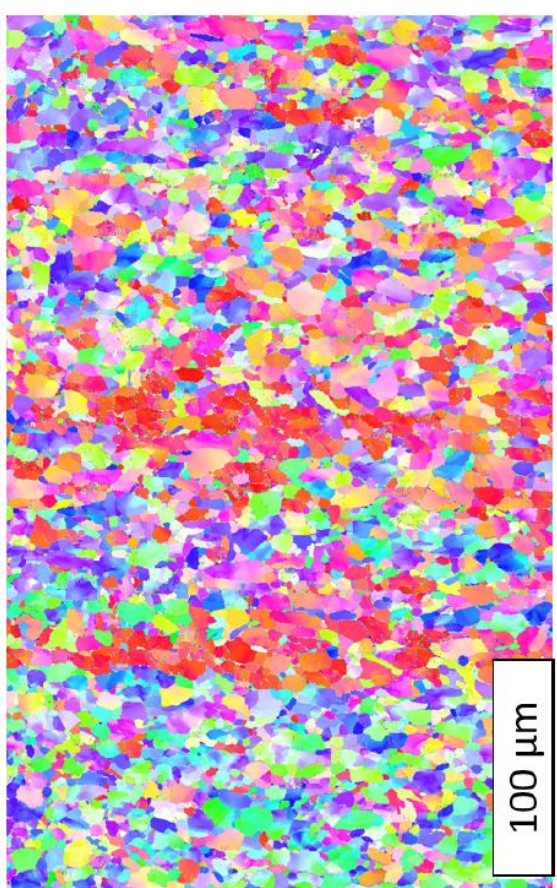

(c)

Figure 1 : EBSD analyses of our two alloys. (a) Crystallographic pole figure of grade 2, (b) the (RD, ND) face associated with (a) and (c) the (ND,TD) face of TA6V ELI with macro-zones. 


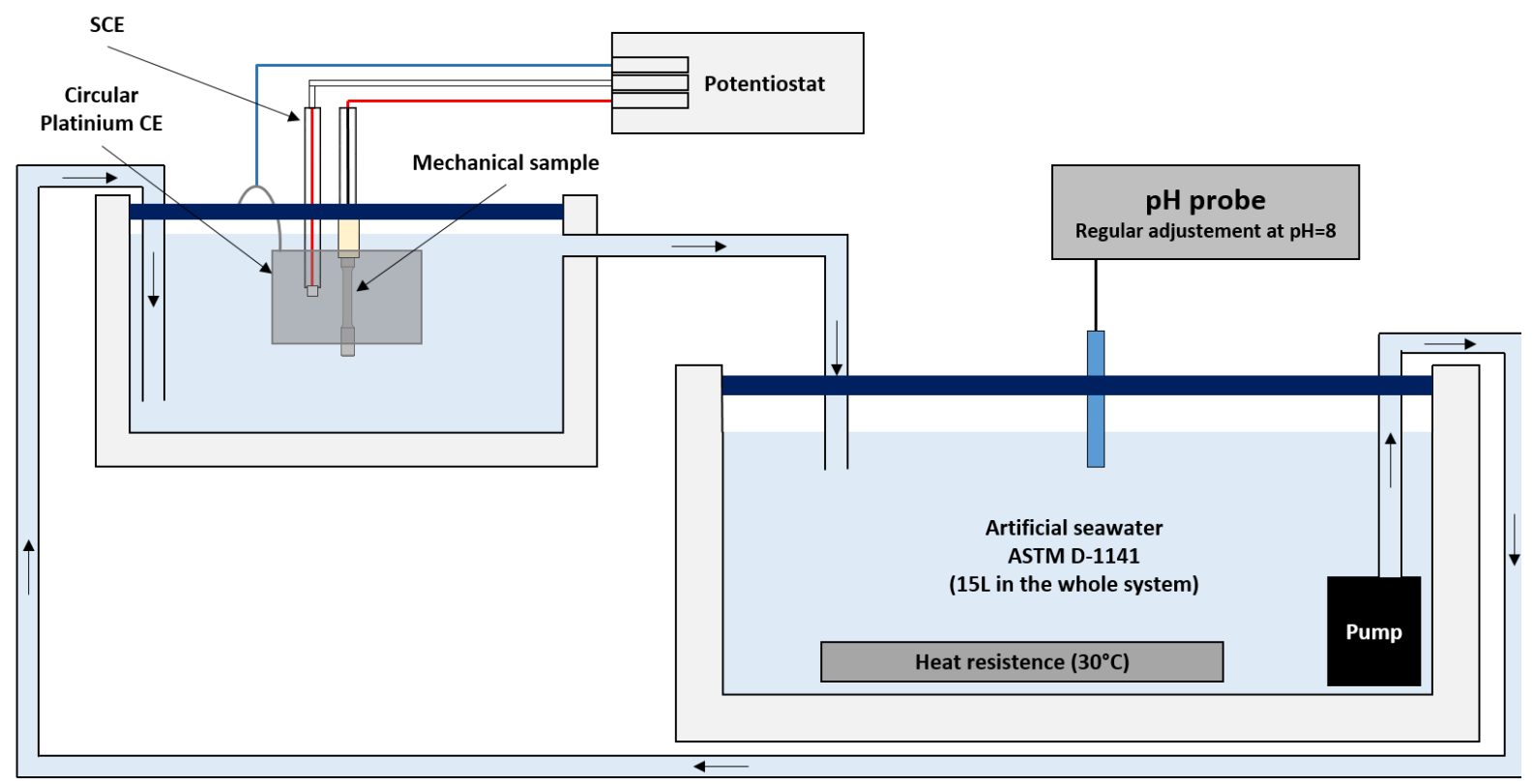

Figure 2 : Hydrogen charging system

\section{Results}

\section{Tensile tests}

Figure 3 presents the conventional tensile tests without notch of grade 2 and grade 23 for different hydrogen concentrations after charging by the electrochemical way. A completely different behaviour is noticed between the two alloys. With add of hydrogen, A\% of grade 2 lowers but the general shape of the curve remains the same. The sample with 492 wppm is different. It has not been tested within de 30 minutes after the hydrogen charging but 10 hours after. We can see that its A\% is higher than it should be, compared to other test, which suggest that the mobile hydrogen promote damage more than hydrides. Looking at Figure 4, we noticed that the fracture mode of smooth samples stays the same even with $825 \mathrm{wppm}$ of hydrogen. The fracture is ductile in "fluting" type. However, the size of the "flutes" seems to decrease with the increase of hydrogen concentration. The orientation of "flutes" and the elliptic surface of fracture are relative to the crystallographic texture. A hydride layer, Figure 5 , is formed with hydrogen absorption on the sub-surface of the samples. The layer is broken perpendicularly to the axis of the tensile test and does not initiate any crack in the $\alpha$-phase itself. Tests of $U$ and V samples are with the same decrease of A\% tendencies. It can be noticed that some hydrides clusters have been seen in all the bulk of the samples independently to the depth considered. U samples show the same fracture mode as smooth samples. The fracture of V samples (V0, 14 show in Figure 6) is somewhat different. The fracture initiates in a ductile way on the surface and spreads brittle in the core of grade 2 . We can also see that the size of the ductile band initiation lower with the increase of hydrogen concentration. 


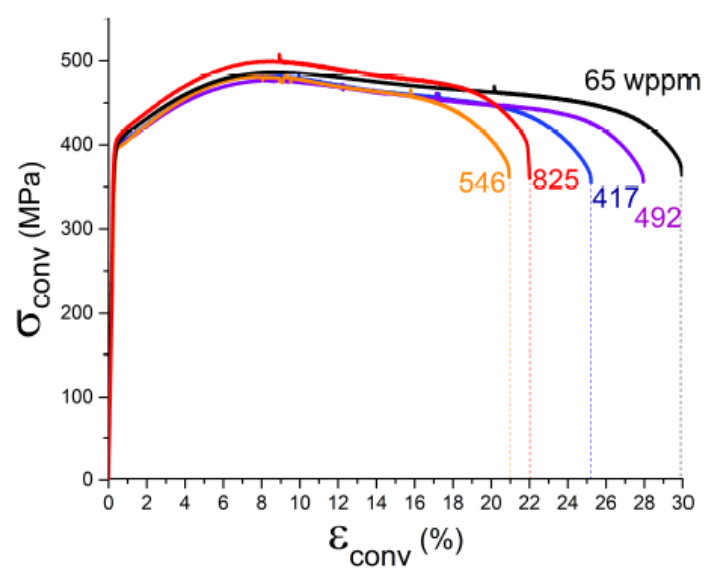

(a)

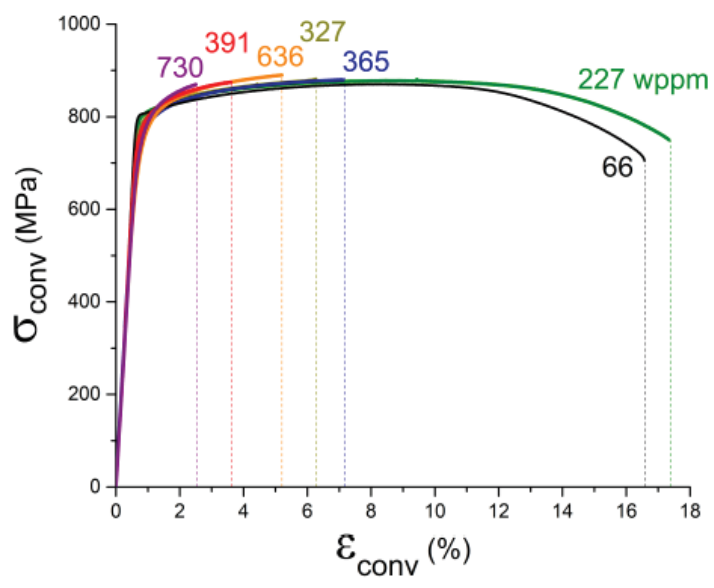

(b)

Figure 3 : Tensile tests with smooth samples without and with hydrogen chargings of (a) grade 2 and (b) grade 23.

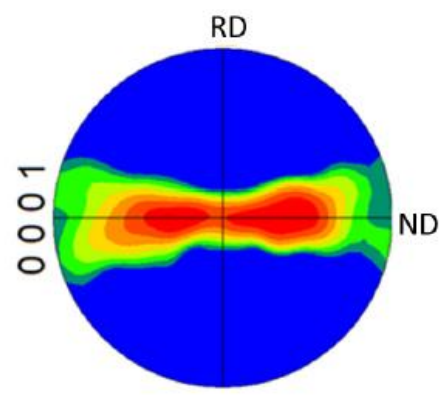

(a)
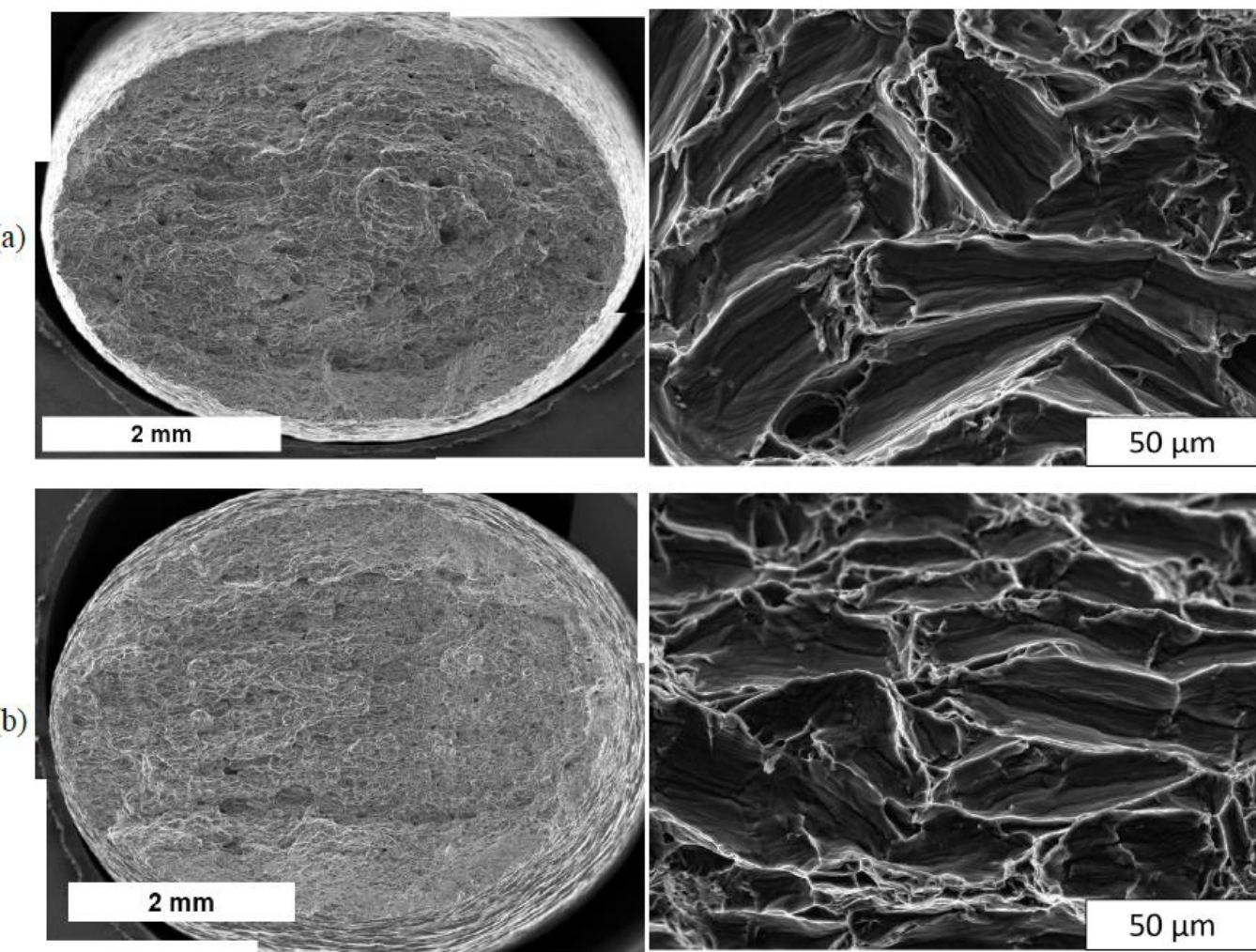

Figure 4 : Pole figure and fracture of smooth grade 2 samples (a) without hydrogen charging and (b) with 825 wppm of hydrogen. 


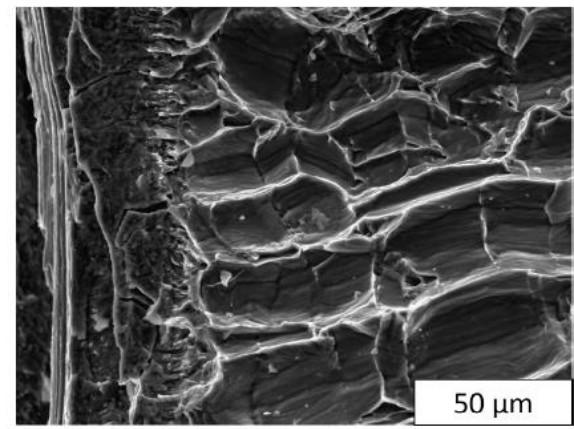

(a)

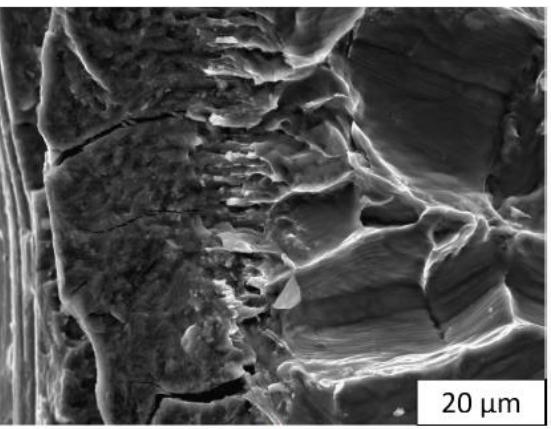

(b)

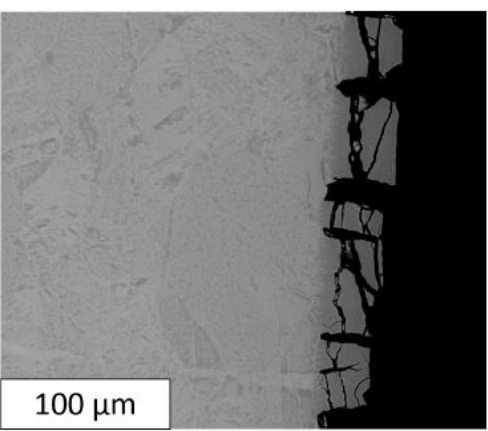

(c)

Figure 5 : Hydride layer on a charged mechanical sample of grade 2 (collected perpendicular to the rolling direction) by $\operatorname{SEM}(a, b)$ on the fracture surface (with the fracture initiation) and (c) by a longitudinal cut.

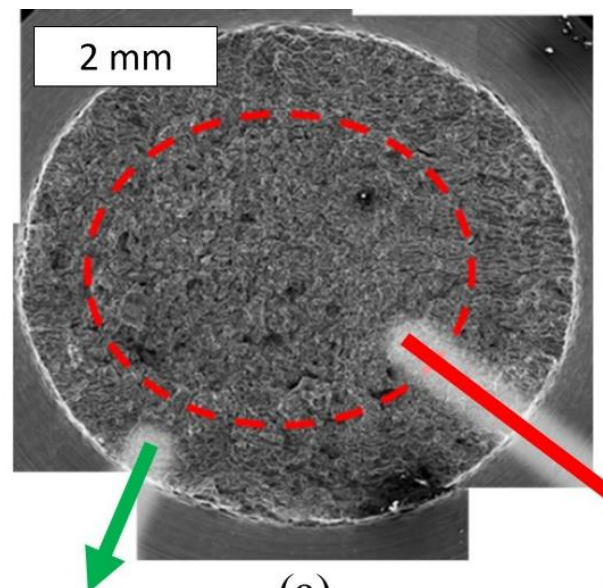

(a)

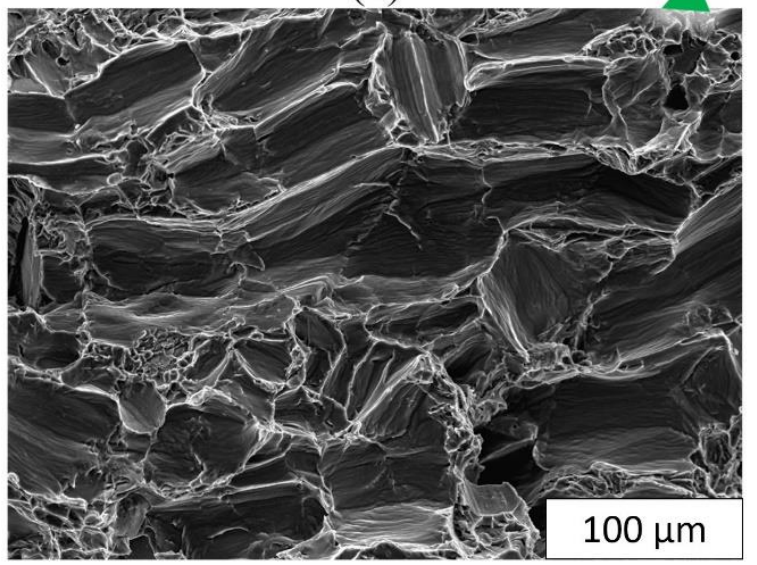

(c)

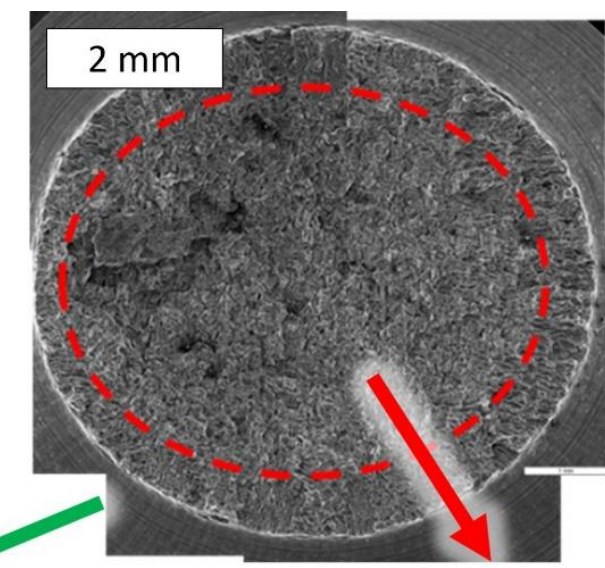

(b)

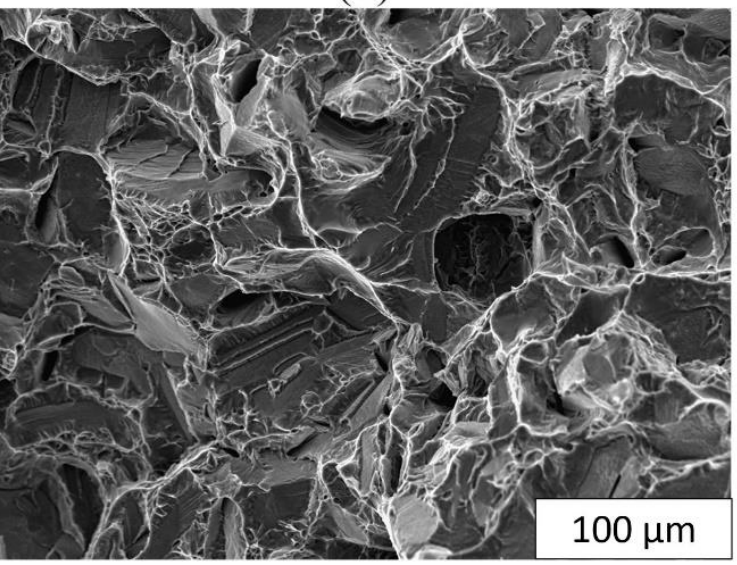

(d)

Figure 6 : Fracture of V0, 14 grade 2 samples (a)without hydrogen and (b) with 500 wppm of hydrogen. (c) Ductile "fluting" fracture initiation on the surface and (d) brittle intern propagation

Grade 23 has a different behaviour (Figure 3). When approximately $300 \mathrm{wppm}$ of hydrogen is exceeded, smooth samples break before the beginning of the necking. Figure 7 shows the fracture surfaces of smooth, U5 and V0.14 samples of grade 23. An impact of macro-zones is noticed with the elliptic shape and on the "fibres" on samples without charging. The fracture mode is ductile. However, these particularities erase when hydrogen exceeds 300 wppm and the samples become brittle as seen on fractures modes. We can also see that the initiation of the fracture 
without hydrogen charging comes from the core of the smooth and U5 samples and from the surface of the sample V0.14. For charged brittle samples, all fractures initiate on the surface. Figure 8 and Figure 9 are SEM analysis on a longitudinal cut of the smooth sample with $636 \mathrm{wppm}$ of hydrogen. It brings out the presence of inter-granular hydrides in the sample. Their presence has been seen on all interfaces in sub-surface, decreasing their number until a small number to the core of this sample; Figure 9 shows the brittle properties of these hydrides. Even in the bulk, some of these hydrides fractured.
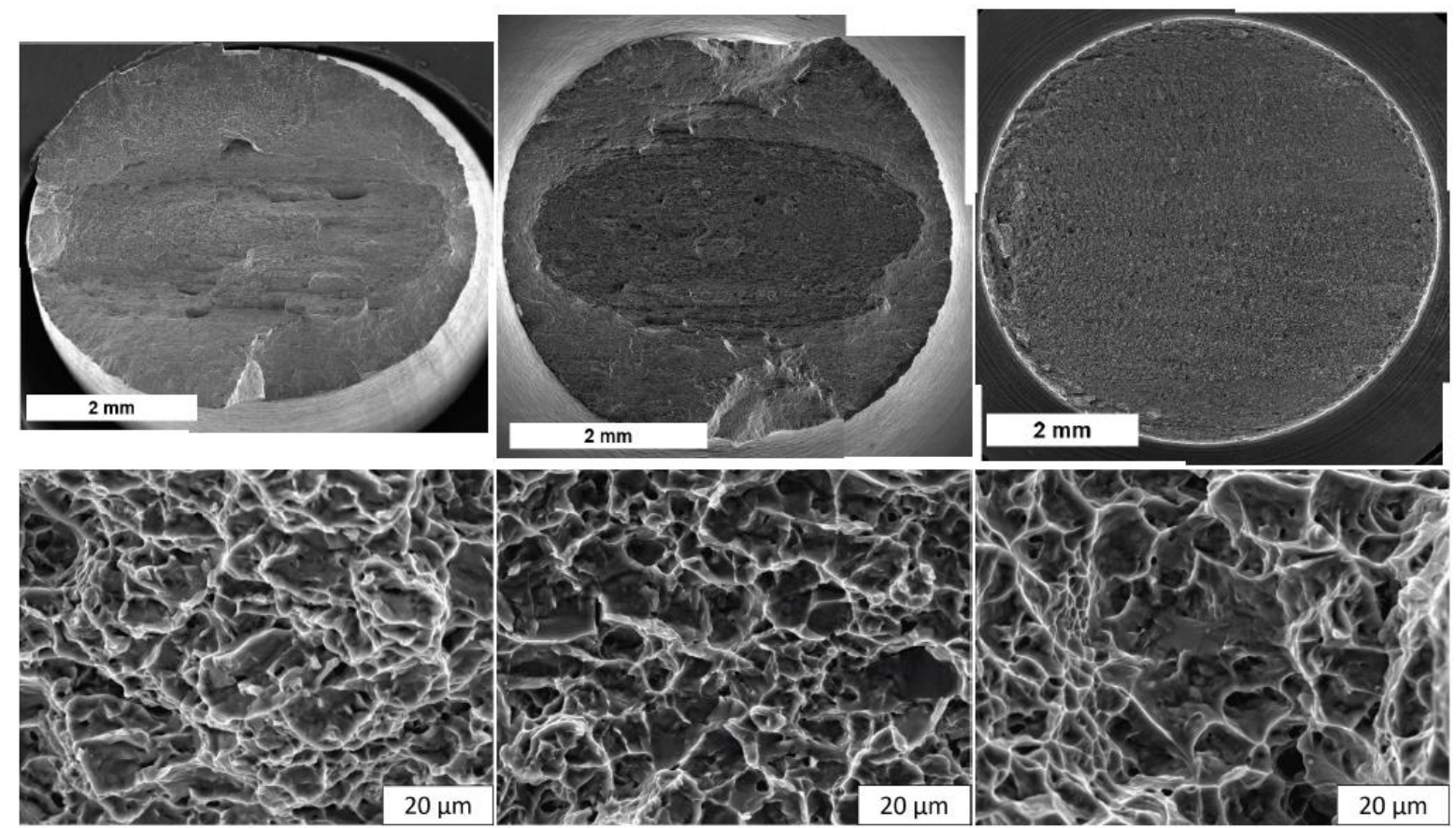

(a)
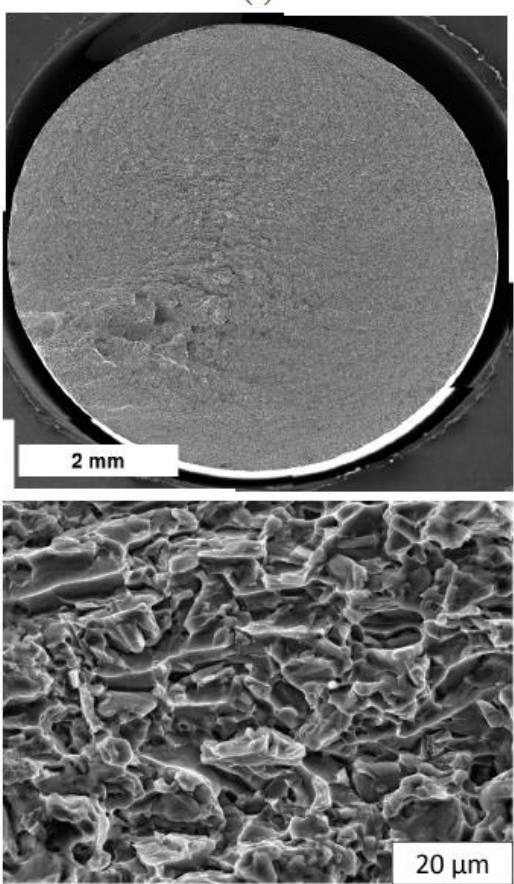

(d) (b)
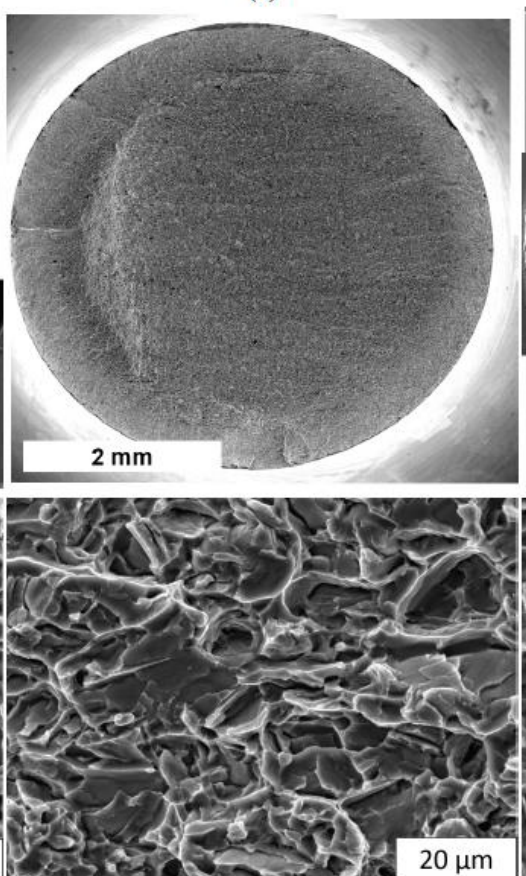

(e) (c)
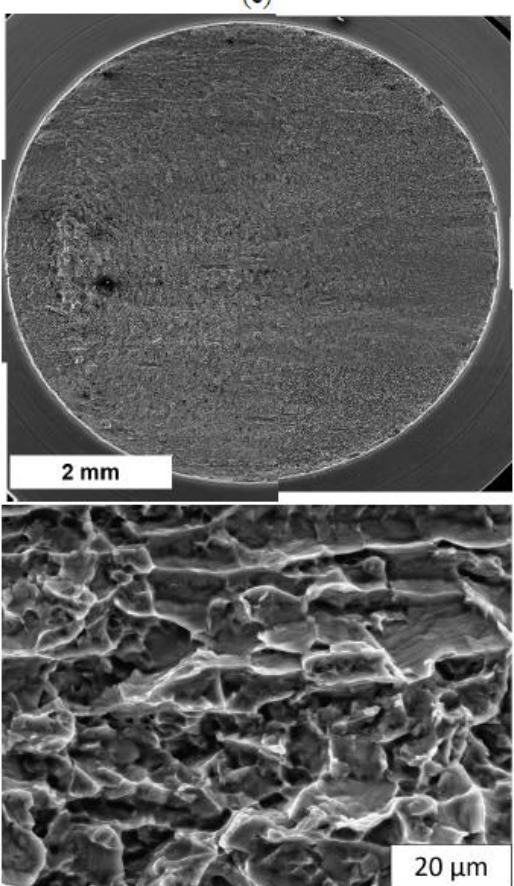

(f)

Figure 7 : Fracture of (a) (d) smooth, (b) (e) U5 and (c) (f) V0, 14 grade 23 samples. (a), (b) and (c) without hydrogen charging. (d) with 365 wppm, (e) 385 wppm and (f) 592 wppm of hydrogen measured on the un-necked zone. 


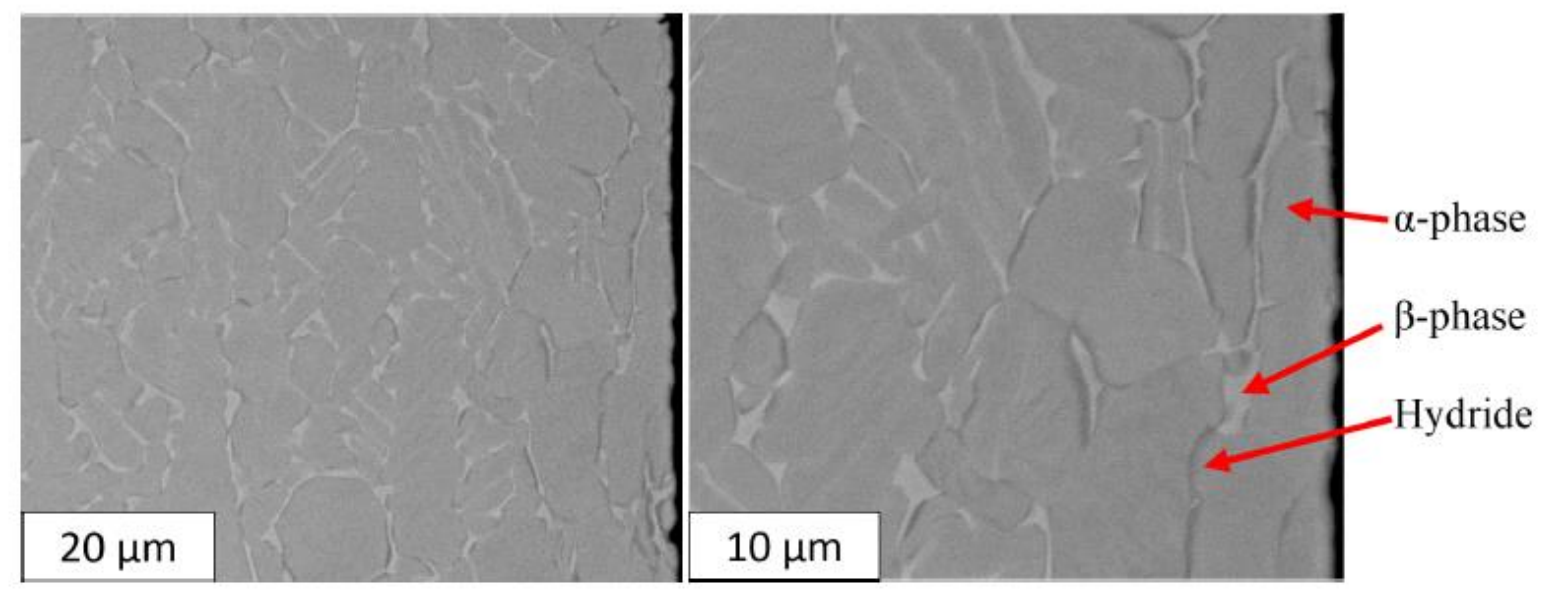

Figure 8 : Hydrides on sub-surface in a charged mechanical sample of grade 23 by SEM-BSE on a longitudinal cut.

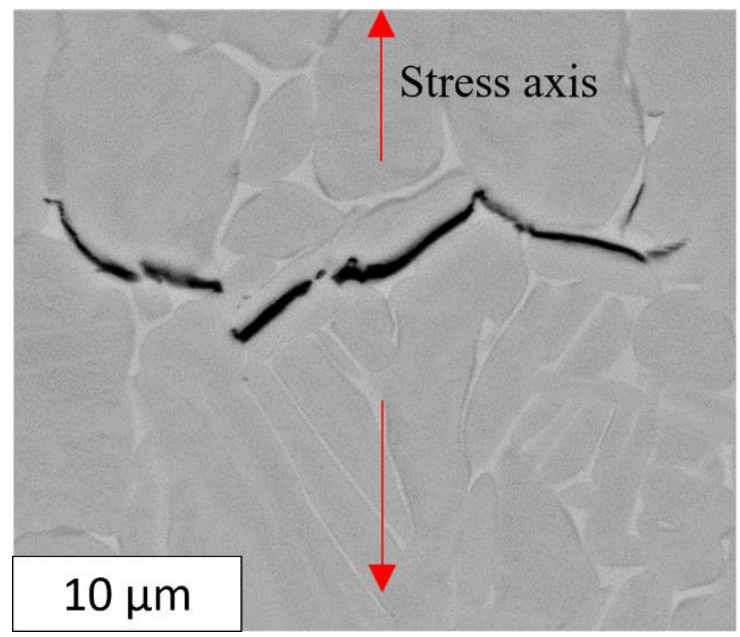

Figure 9 : Fracture in intergranular hydrides observed at $1 \mathrm{~mm}$ depth and $150 \mu \mathrm{m}$ below the fracture of a smooth sample of grade 23 with 636 wppm of hydrogen.

\section{Numerical approach}

Figure 10 presents the mesh used for the modelling Smooth, U5 and V0.14 samples and maps representing the localisation of hydrostatic stress $\sigma_{\mathrm{m}}$ and equivalent plastic strain $\varepsilon_{\mathrm{peq}}$ on a longitudinal section at the last modelling point (before fracture) without hydrogen charging. We can see that $\sigma_{\mathrm{m}}$ and $\varepsilon_{\mathrm{peq}}$ are maximum in the core of the smooth samples and on the surface of the V samples, which correspond to the fracture initiations seen on the precedent part. Figure 11 presents the numerical stress/strain curves compared to the experimental ones on both alloys. We modelled until the beginning of the fracture and does not considered the fracture propagation. Because this is an isotropic low, the reduction of sample section obtained is between the values we have observed experimentally for the two axes of the ellipse (Figure 4 and Figure 7). An anisotropic plastic criterion will be applied in future work.

Figure 12 shows the local conditions of fracture of grades 2 and 23 in term of $\sigma_{\mathrm{m}}$ and $\varepsilon_{\text {peq. }}$. We see the differences between grade 2 which can support a higher local equivalent plastic strain and grade 23 which can support nearly two times higher hydrostatic stress than grade 2. The same approach with each type of sample was used for samples with hydrogen charging and presented in Figure 12. Grade 2 with 500 wppm presents a low decrease of local mechanical properties even if hydrides have been detected as a layer on surface and sub-surface as presented in 
Figure 5 and also in all the bulk of the sample. Hydrides are presents inside grade 23 when the local solubility limit of $\beta$-phase is exceeded (higher than $300 \mathrm{wppm}$ approximately). Some hydrides have been observed even in the middle of a smooth sample which measured $6.35 \mathrm{~mm}$ of diameter, so hydrogen have penetrate mare than $3 \mathrm{~mm}$ deep in the alloy. It is clearly seen that hydrides lower the mechanical strength of this alloy by fracturing prematurely with a limit of the plastic deformation of approximately $5 \%$.

(a)
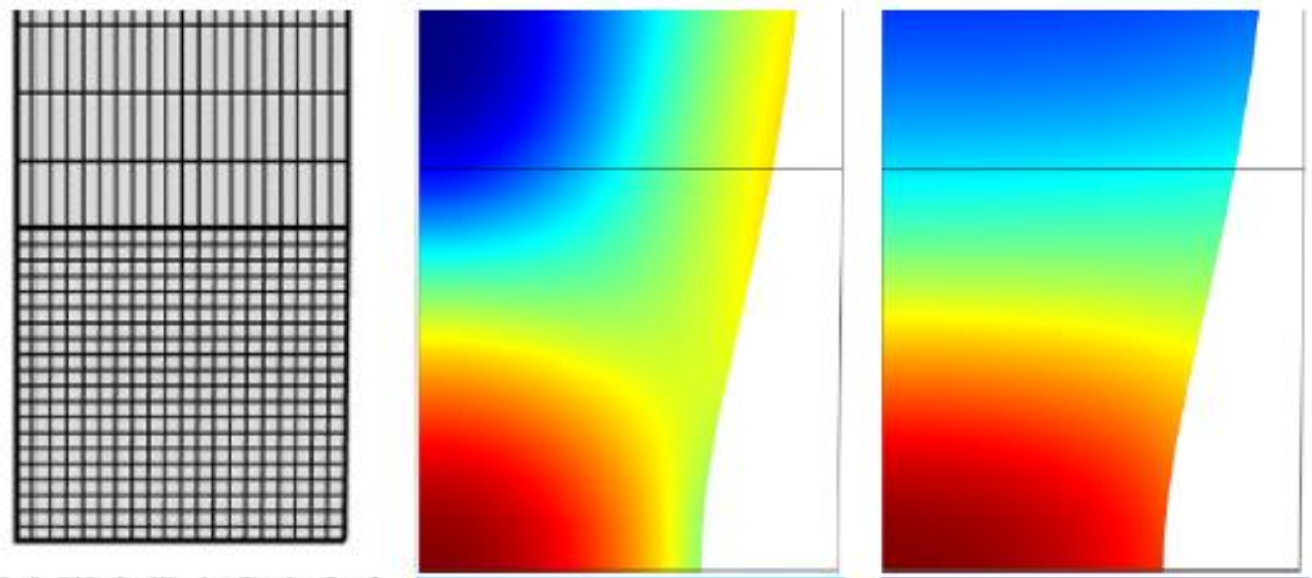

(b)
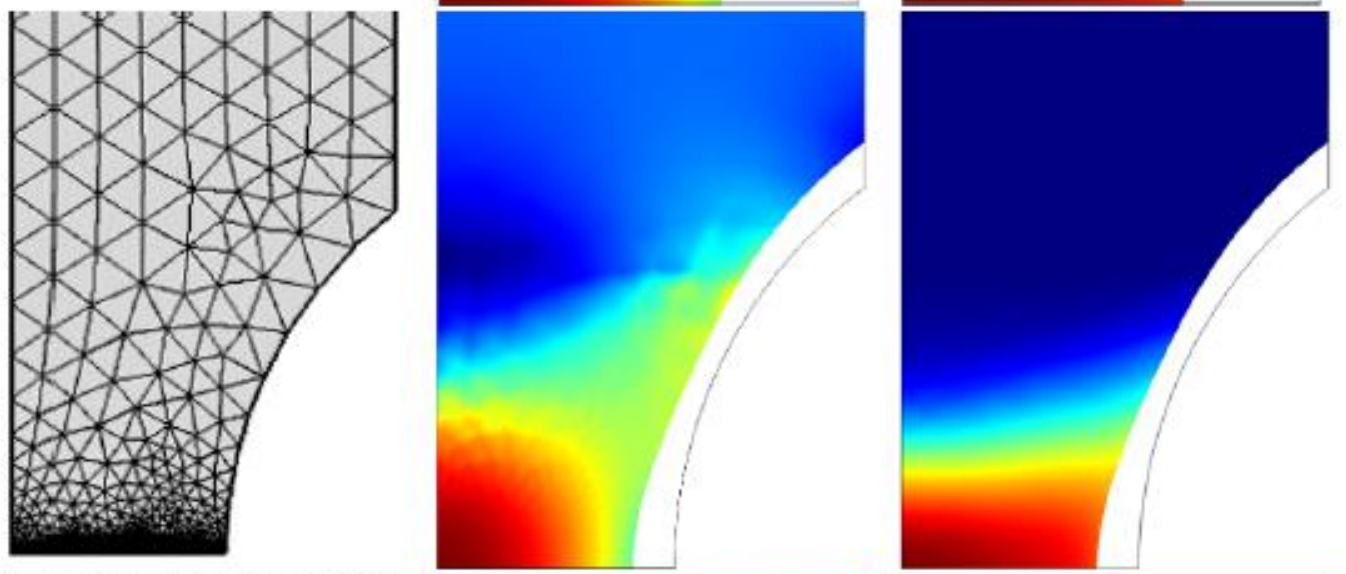

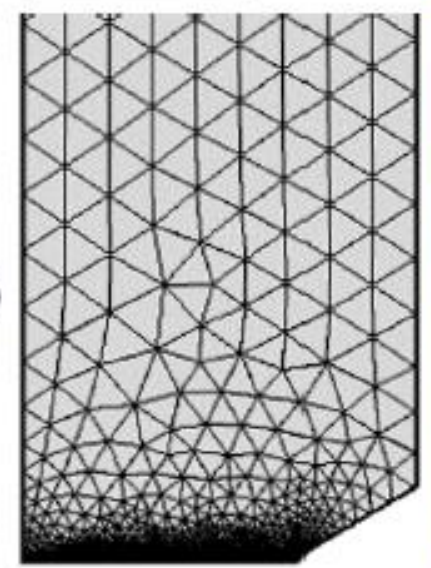

Mesh

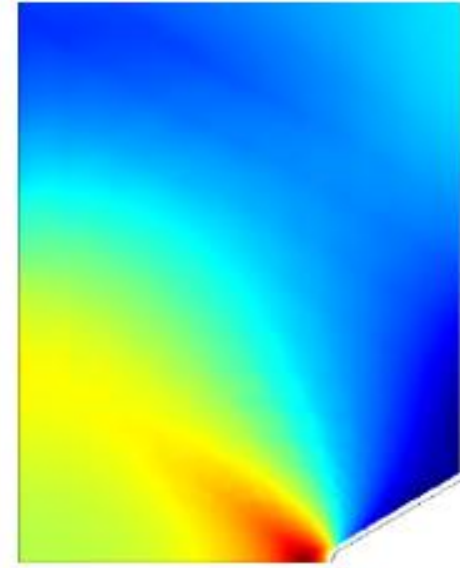

Hydrostatic stress

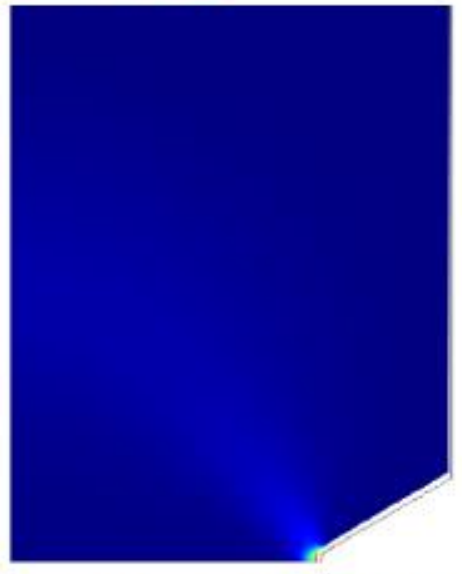

Equivalent plastic strain

Figure 10 : Mesh of modelled (a) smooth, (b) U5 and (c) V0, 14 samples and localisation of hydrostatic stress and equivalent plastic strain as a function of the radius at the last point of modelling. 


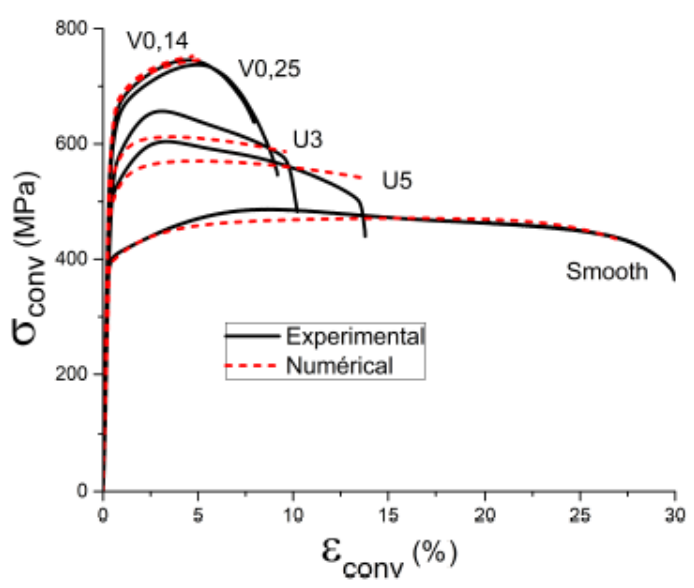

(a)

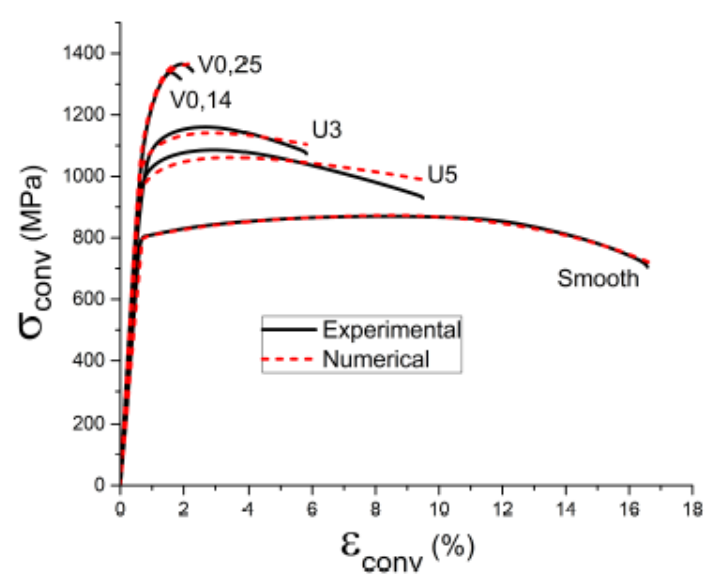

(b)

Figure 11 : Experimental and numerical conventional stress/strain curves of (a) grade 2 and (b) grade 23.

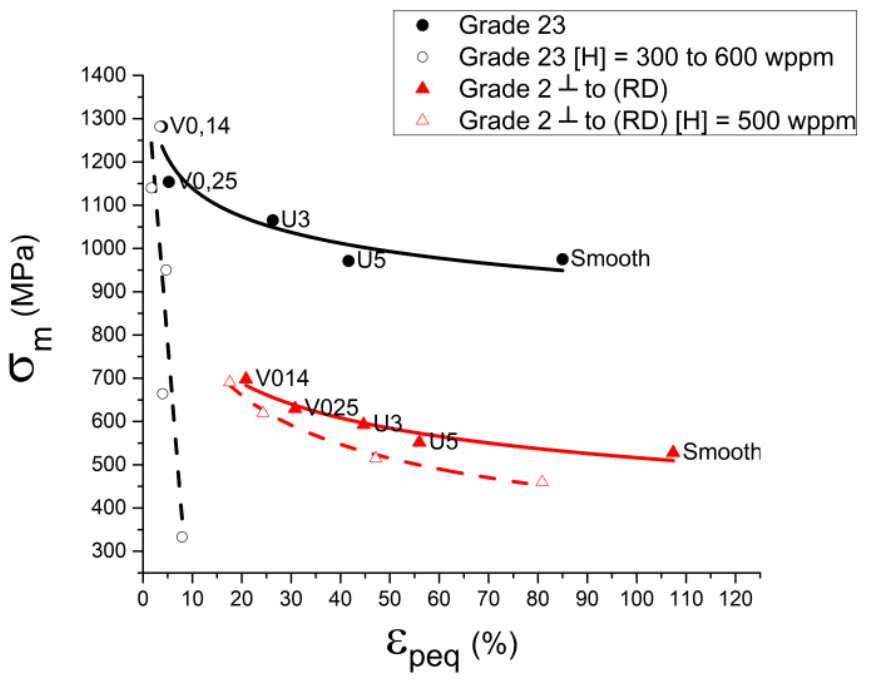

Figure 12 : Local conditions of fracture of grades 2 and 23 in term of hydrostatic stress and equivalent plastic strain

\section{Discussion}

Grade 2 is a polycrystalline metal where grain-boundaries promote hydrogen diffusion. Hydrogen charged by the electrochemical way decreases the ductility of grade 2 but doesn't change its fracture mode (fluting mode). This small reduction of ductility has been illustrated in Figure 12. This single-phase alloy formed a hydride layer on its surface during hydrogen charging and we observed that this layer break during tensile tests without initiating any fracture in the $\alpha$-phase itself and without modifying the fracture mode of samples. Some hydrides clusters have also been identified in all the bulk of the alloy. Hydrides are generally known to cause embrittlement but it has been shown that they can be ductile dependently to their crystallographic arrangement with the matrix during their formation [5]. It has also been shown that hydrides clusters can promote void nucleation on grade 2 and have a moderate impact on the fracture [2]. Embrittlement of grade 2 was shown in precedent works [6] but with gaseous charging at $475^{\circ} \mathrm{C}$ and higher hydrogen concentration (600 to $\left.3000 \mathrm{wppm}\right)$, so hydrogen diffused in a great content in all the alloy and formed a lot of hydrides in all the bulk with a large density of interfaces. In our study, most of the hydrides consist of the surface and sub-surface layer even if there is some in the bulk. Because of these observations, we will continue our study with stronger concentrations of hydrogen and with homogenisation by thermal treatments. Past studies show a similar fracture of titanium (flutes) with and without hydrogen [7], [3]. 
Sections of deformed samples show that these shapes form at the intersection of slip bands or deformation twins [8]. This morphology is induced by the low symmetry of the $\alpha$-phase hexagonal structure where prismatic glides are predominant. Sample testing after a long aging time (492 wppm) Figure 3 highlight a lower loss of ductility than the other hydrogen charging samples which question the respective impact of hydrides and solute hydrogen on damage.

Grade 23 is a two-phase alloy and react very differently to the addition of hydrogen. Hydrides forms after a hydrogen concentrations of approximately $300 \mathrm{wppm}$. Considering that hydrogen is absorbed in $\beta$-phase during the charging and hydrides formed after the saturation of this phase which is $6 \%$ of the structure, the limit of 300 wppm in the alloy is the hydrogen solubility limit of $5000 \mathrm{wppm}$ in the $\beta$-phase [9]. Immediately after the first hydrides precipitations, mechanical properties of this alloy falls (Figure 12). The fracture becomes brittle and initiate on the sub-surface regardless of the sample type. The propagation follows hydrides, which are principally located on the grain boundaries ( $\alpha / \beta$ interfaces and $\alpha$ boundaries).

Figure 12 is the diagrams representing the type of fracture as a function of the stress concentration and the hydrogen concentration measured on the un-necked zone (average hydrogen content) of the samples after the tensile test. All samples tested have been represented. As we explained, there is not changes of fractures type in grade 2 but a reduction of "flutes" size and decrease of A\%. Grade 23 present a limit after when it becomes brittle. This limit between ductile and brittle fracture is here relatives to samples shape and size. The variation observed in the picture is mostly caused by the way we measured the hydrogen concentration. The different shapes of samples can modify locally the charging conditions and the local concentration of hydrogen. For future work, to access this last concentration, we will perform FEM calculations with diffusion model associated to the mechanical model

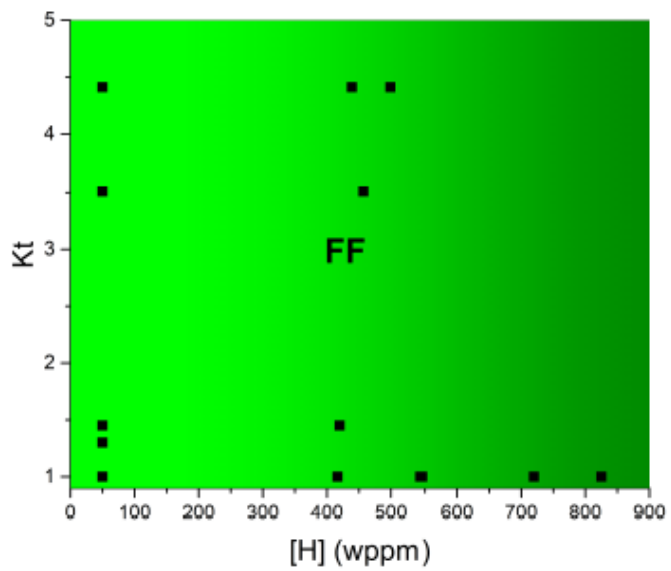

(a)

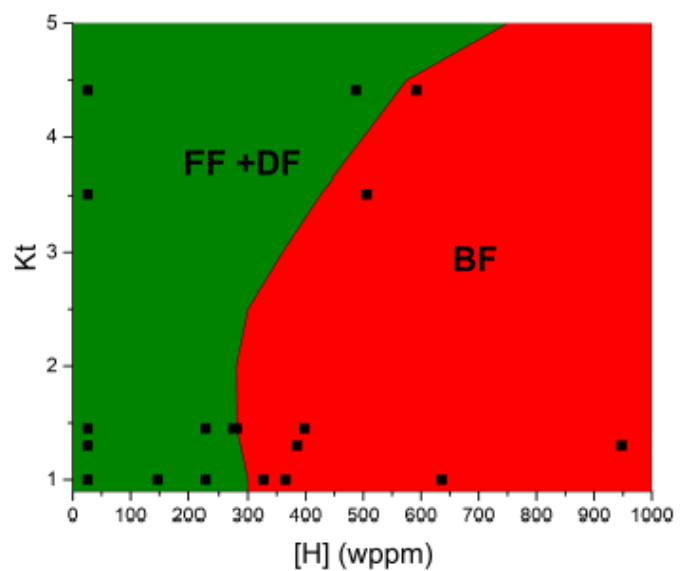

(b)

Figure 13 : Fracture type as a function of $\mathrm{Kt}$ and hydrogen concentration (measured on the un-necked zone of samples) of (a) grade 2 and (b) grade 23. Squares represents the mechanical samples tested. FF is fluting fracture, DF is dimple fracture and $\mathrm{BF}$ is brittle fracture

\section{Conclusion}

Hydrogen charged by cathodic polarization appeared to have a moderate impact on grade 2 reducing the ductility for hydrogen content lower than $500 \mathrm{wppm}$. Sub-surface hydride layer breaks during tensile tests without initiate the fracture of the sample and diffusible hydrogen could be at the origin of the reduction of mechanical properties. In opposite, hydrogen leads to strong embrittlement of grade 23 for hydrogen content higher than 300 wppm when hydrides forms at the interfaces of $\alpha$-phase and $\beta$-phase and at the $\alpha$ grain boundaries. 


\section{$\underline{\text { References }}$}

[1] Y. Combres « Métallurgie et recyclage du Titane et de ses alliages », Techniques de l'ingénieur, M2355 V1, 10 juin 1997.

[2] J. Huez, X. Feaugas, A.L. Helbert, I. Guillot, and M. Clavel "Damage Process in Commercially Pure aTitanium Alloy without (Ti40) and with (Ti40-H) Hydrides", Metallurgical and materials transactions A, Volume 29A, June 1998, p.1615-1628.

[3] U. Bathini, T.S. Srivatsan, A. Patnaik, and T. Quick "A Study of the Tensile Deformation and Fracture Behavior of Commercially Pure Titanium and Titanium Alloy: Influence of Orientation and Microstructure", Journal of Materials Engineering and Performance, Volume 19(8) November 2010, p.1172-1182.

[4] Li Miaoquan, Zhang Weifu, Zhu Tangkui, Hou Hongliang, Li Zhiqiang « Effect of Hydrogen on Microstructure of Ti-6Al-4V Alloys” Rare Metal Materials and Engineering, 2010, 39(1): 0001-0005.

[5] E. Conforto, I. Guillot, X. Feaugas. "Solute hydrogen and hydride phase implications on the plasticity of zirconium and titanium alloys: a review and some recent advances" 2017, Phil. Trans. R. Soc. A.

[6] C.L. Briant, Z.F. Wang, N. Chollocoop "Hydrogen embrittlement of commercial purity titanium", Corrosion Science 44 (2002) 1875-1888.

[7] A.L. Pilchak and J.C. Williams "Crystallography of Fluted Fracture in Near-a Titanium Alloys" Metallurgical and materials transactions A, Volume 41A, January 2010, p.22-24.

[8] R.H. Van Stone, J. R. Low, Jr., and J. L. Shannon, Jr. "Investigation of the Fracture Mechanism of Ti-5AI2.5Sn at Cryogenic Temperatures" Metallurgical and materials transactions A, Volume 9A, April 1978, p.539552.

[9] A. Poloni, A. Oudriss, J. Creus, C. Berziou, E. Conforto, C. Savall, S. Frappart, T. Millot, A. Mathis, X. Feaugas "Hydrogen absorption and hydride formation in pure titanium T40 (grade 2) and TA6V ELI (grade 23) under cathodic polarization in artificial seawater" proceeding, June 10-14, 2019, Nantes - France, Titanium2019. 